\title{
Efectos del Trichoderma sp. sobre el crecimiento y desarrollo de la arveja (Pisum sativum L.)
}

\section{Effects of Trichoderma sp. on the pea (Pisum sativum L.) growth and development}

Fecha recepción: 28 de mayo de 2013

Fecha Aprobación: 11 de noviembre de 2013
David Fernando Camargo-Cepeda1', Edwin Ricardo Ávila²

\section{RESUMEN}

Se estima que el cultivo de arveja en Colombia genera alrededor de 2,3 millones de jornales y unos 15.000 empleos directos; de él dependen más de 26.000 productores (1). Ante la ausencia de alternativas de producción, el agricultor ha recurrido tradicionalmente a la aplicación de productos de síntesis química, práctica que cada vez se encuentra más restringida por razones económicas y ecológicas (2); por esto, se hace necesario encontrar nuevos modelos que contribuyan a mejorar la calidad de vida de los productores. El trabajo determinó los efectos de la aplicación de Trichoderma sp. sobre el crecimiento y desarrollo de la arveja. Se realizó el aislamiento de la cepa nativa de Trichoderma sp. a partir de suelo proveniente de cultivos de arveja; luego se procedió a hacer las diluciones tanto de Trichoderma sp. nativa como de la comercial; se inocularon las plantas y se realizó la medición de las variables de crecimiento y desarrollo.

\begin{abstract}
It is estimated that the cultivation of pea generates about 2.3 million wages and about 15,000 direct jobs, from which depend more than 26,000 producers (1). In the absence of alternative production, the farmer has traditionally made use of synthetic chemical products, practice which is becoming more restricted for economic and ecological reasons, affecting negatively the population (2). So we need to find new production models that contribute to improve the life quality of the farmers who depend on the crop. This work determined the effects of the Trichoderma sp. application on the pea growth and development. The Trichoderma sp. native strain was isolated from soil of the pea crop.Then there were carried out Trichoderma sp. dilutions, in both the native and commercial plants, Afterwards the plants were inoculated and measured the variables of growth, development and germination, such as plant height, number of stems, disease incidence, leaf
\end{abstract}

Profesional independiente. Correo Electrónico: davidcam2012@gmail.com

Profesional independiente. 
La aplicación de Trichoderma sp. comercial en el cultivo de arveja mejora notablemente su crecimiento y desarrollo, influyendo en variables fisiológicas como germinación, área foliar, peso seco y fresco de la raíz, peso seco y fresco de la parte aérea, y longitud de raíz.

Palabras clave: Arveja, Control de plagas, Fungicidas biológicos, Trichoderma sp. area, fresh weight of root and aerial part dry weight of root and aerial part, root length. Therefore the application of Trichoderma in commercial pea crop, significantly improves the growth and development of this culture, influencing its physiological variables such as germination, leaf area, root dry weight, root fresh weight, dry weight of the aerial parts, fresh weight shoot and root length.

Key Words: Pea, Trichoderma sp., Biologic Fungi, Infections Control. 


\section{INTRODUCCIÓN}

La arveja (Pisum sativum L), después del fríjol, es la leguminosa de mayor importancia en Colombia; su cultivo ha sido un factor estabilizador de la economía de los pequeños productores de las zonas andinas y ha contribuido a la seguridad alimentaria (1); es cultivada en 14 departamentos, de los cuales, Cundinamarca y Boyacá son los principales productores $(2,3)$.

La cosecha nacional está orientada a satisfacer la demanda de producto en fresco, mientras que la demanda del producto en seco se cubre con importaciones provenientes de Canadá (4). El material sembrado, predominante, es el cultivar Santa Isabel, que satisface los requerimientos del mercado y ocupa casi toda el área del país sembrada en arveja. La incidencia y la persistencia de plagas y enfermedades en el cultivo disminuyen los rendimientos, elevan los costos de producción y ambientales y reducen las áreas de viabilidad del cultivo, limitando el crecimiento en términos de área cultivada, pese a las posibilidades de mercado (4).

Se ha encontrado que especies de Trichoderma actúan como hiperparásitos competitivos que producen metabolitos antifúngicos y enzimas hidrolíticas que causan cambios estructurales a nivel celular del patógeno, tales como vacuolización, granulación, desintegración del citoplasma y lisis celular (5). Además del efecto biocontrolador de patógenos, se ha reportado que la inoculación de $T$. harzianum aporta otros beneficios a las plantas: a través de la descomposición de materia orgánica, libera nutrientes en formas inmediatamente disponibles (6, 7); por medio de la actividad solubilizadora de fosfatos $(7,8,9)$, promueve el crecimiento y el desarrollo de los cultivos, produciendo metabolitos que estimulan los procesos de desarrollo vegetal (10), y la capacidad de multiplicarse en el suelo y colonizar las raíces de las plantas libera factores de crecimiento (auxinas, giberelinas y citoquininas) que estimulan la germinación y el desarrollo de las plantas (11). T. harzianum ha sido destacado como promotor del crecimiento vegetal en cultivos de berenjena, fríjol, café, tomate, papa y especies forestales, entre otros $(12,13)$. Por lo anterior, es conveniente determinar los efectos de la aplicación de Trichoderma sp. sobre el crecimiento y el desarrollo de la arveja.

\section{MATERIALES Y MÉTODOS}

Sitio de estudio. En la Universidad Pedagógica y Tecnológica de Colombia, sede central, Tunja, ubicada a $5^{\circ} 32^{\prime}$ 05" latitud Norte y $73^{\circ} 21^{\prime} 52^{\prime \prime}$ longitud oeste, con unas condiciones geoclimáticas de $553 \mathrm{~mm}$ de precipitación, temperatura media de $13{ }^{\circ} \mathrm{C}$ y altura de 2820 msnm.

Para este trabajo de investigación se utilizó el diseño Completamente al Azar (DCA), con 13 tratamientos y 3 repeticiones. La unidad experimental estuvo representada por 1 bolsa de polipropileno con $2 \mathrm{~kg}$ de suelo y una planta por bolsa. Para el análisis estadístico se utilizó el programa SAS versión 9.2.

Tabla I. Tratamientos del proyecto

\begin{tabular}{|c|c|}
\hline Tratamiento & Descripción \\
\hline $\mathrm{T}_{1}$ & $\begin{array}{l}\text { Trichoderma nativa en concentración } \\
1 * 10^{3} \text { esporas } / \mathrm{ml}\end{array}$ \\
\hline $\mathrm{T}_{2}$ & $\begin{array}{l}\text { Trichoderma nativa en concentración } \\
1 * 10^{4} \text { esporas } / \mathrm{ml}\end{array}$ \\
\hline $\mathrm{T}_{3}$ & $\begin{array}{l}\text { Trichoderma nativa en concentración } \\
1 * 10^{5} \text { esporas } / \mathrm{ml}\end{array}$ \\
\hline $\mathrm{T}_{4}$ & $\begin{array}{l}\text { Trichoderma nativa en concentración } \\
1 * 10^{6} \text { esporas } / \mathrm{ml}\end{array}$ \\
\hline $\mathbf{T}_{5}$ & $\begin{array}{l}\text { Trichoderma nativa en concentración } \\
1 * 10^{7} \text { esporas } / \mathrm{ml}\end{array}$ \\
\hline$T_{6}$ & $\begin{array}{l}\text { Trichoderma nativa en concentración } \\
1 * 10^{8} \text { esporas } / \mathrm{ml}\end{array}$ \\
\hline $\mathrm{T}_{7}$ & Testigo sin inocular \\
\hline $\mathrm{T}_{8}$ & $\begin{array}{l}\text { Trichoderma comercial en } \\
\text { concentración } 1 * 10^{3} \text { esporas } / \mathrm{ml}\end{array}$ \\
\hline$T_{9}$ & $\begin{array}{l}\text { Trichoderma } \quad \text { comercial en } \\
\text { concentración } 1 * 10^{4} \text { esporas } / \mathrm{ml}\end{array}$ \\
\hline$T_{10}$ & $\begin{array}{l}\text { Trichoderma comercial en } \\
\text { concentración } 1 * 10^{5} \text { esporas } / \mathrm{ml}\end{array}$ \\
\hline $\mathrm{T}_{11}$ & $\begin{array}{l}\text { Trichoderma comercial en } \\
\text { concentración } 1 * 10^{6} \text { esporas } / \mathrm{ml}\end{array}$ \\
\hline$T_{12}$ & $\begin{array}{lcc}\text { Trichoderma } & \text { comercial en } \\
\text { concentración } 1 * 10^{7} \text { esporas } / \mathrm{ml}\end{array}$ \\
\hline$T_{13}$ & $\begin{array}{l}\text { Trichoderma comercial en } \\
\text { concentración } 1 * 10^{8} \text { esporas } / \mathrm{ml}\end{array}$ \\
\hline
\end{tabular}

Aislamiento del Trichoderma nativo. Para el aislamiento del Trichoderma nativo se utilizó el protocolo de Cervantes et al. (14). A partir de una muestra representativa de suelo proveniente de un 
cultivo de arveja se tomaron 10 gramos de suelo y se suspendieron en 95 mililitros de solución salina $(\mathrm{NaCl}$ al $0.85 \%$ ), y se agitaron durante 20 minutos, con el fin de desagregar las partículas del suelo y obtener una suspensión homogénea. Luego de agitar se dejó reposar la solución por un tiempo, para permitir la precipitación de las partículas de suelo. Luego se procedió a preparar una serie de diluciones en base 10 , hasta $10^{-6}$. Posteriormente, de cada una de estas soluciones se tomó $1 \mathrm{~mL}$ y se llevó a cajas de Petri con medio de cultivo PDA. De los crecimientos se identificaron las colonias de Trichoderma sp. Las cepas del hongo Trichoderma sp. que se aislaron del suelo se cultivaron en el medio Papa-Dextrosa-Agar (PDA) durante veinte días a temperatura de $25{ }^{\circ} \mathrm{C}$; posteriormente se procedió a seleccionar la cepa más infectiva (la que mostró mayor crecimiento en el medio) y se cultivó en medio arroz nutritivo.

Inoculación de la semilla. Para inocular las semillas con la cepa de Trichoderma sp. se adaptó el protocolo propuesto por Lara (15): se sumergieron las semillas de arveja en soluciones con las concentraciones propuestas anteriormente $\left(10^{3}, 10^{4}, 10^{5}, 10^{6}, 10^{7}, 10^{8}\right.$ esporas $\left./ \mathrm{ml}\right)$ durante 24 horas, y posteriormente se sembraron en las bolsas que contenían $2 \mathrm{~kg}$ de suelo. Al momento de la siembra el suelo fue inoculado con la suspensión de Trichoderma comercial y nativo en las diferentes concentraciones $\left(10^{3}, 10^{4}, 10^{5}, 10^{6}, 10^{7}, 10^{8}\right.$ esporas $/ \mathrm{ml}$ ) en el fondo del agujero que se abrió para depositar la semilla, colocando 10 mililitros por planta a las diferentes concentraciones. En total se realizaron tres aplicaciones: a la siembra, a los 30 y a los 60 días después de la siembra.

Siembra de la semilla. El suelo para la siembra provino de terrenos utilizados para el cultivo de arveja. La fertilización se llevó a cabo mediante la aplicación de $50 \mathrm{~kg}^{*} \mathrm{ha}^{-1}$ de Rafos (12-24-12 + menores) al momento de la siembra, incorporado al suelo, para simular las aplicaciones realizadas por los agricultores de la zona. Las semillas fueron adquiridas a un distribuidor autorizado; se sembraron 4 semillas por bolsa, para realizar un raleo posterior a la germinación. Las labores de riego se llevaron a cabo según las necesidades del cultivo.

\section{Toma de datos}

Germinación de la semilla. Fue determinada mediante la relación entre el número de semillas sembradas y el número de semillas que germinaron dentro de los 8 primeros días de cultivo.

Altura de la planta. Se determinó realizando una medición desde el cuello de raíz hasta el meristemo más distal de la parte aérea de la planta; esta medición se llevó a cabo cada 8 días.

Número de tallos. Tomando como base el tallo principal de la planta se determinó el número de tallos secundarios emergidos por planta; esta medición se llevó a cabo cada 8 días.

Incidencia de enfermedades. La incidencia de enfermedades se determinó mediante análisis comparativo con los patrones preestablecidos para cada enfermedad en particular; esta medición se llevó a cabo cada 8 días.

Longitud de raíz. Se determinó tomando la planta y midiendo desde el cuello de raíz hasta el extremo más distal de la raíz de la planta; esta medición se llevó a cabo cada 30 días.

Área foliar. Se determinó mediante el uso de un medidor electrónico de área foliar tomando cada uno de los foliolos de la plantas de estudio y pasándolos por la máquina para determinar su área, luego, la sumatoria del área de los foliolos de cada planta nos dio como resultado el área foliar de la planta.

Peso fresco de la raíz y parte aérea. Se tomó la planta y se seccionó por el cuello de la raíz, para posteriormente, y mediante el uso de una balanza electrónica, tomar el registro del peso de cada una de las partes.

Peso seco de raíz y parte aérea. Se tomaron los tejidos seccionados por el cuello de la raíz y se Ilevaron a una mufla a $100{ }^{\circ} \mathrm{C}$, durante 72 horas, para luego de esto determinar el peso seco de cada una de las partes. 


\section{RESULTADOS Y DISCUSIÓN}

Germinación. Se destaca el efecto de T5, T6, T12 y T13, ya que presentan diferencia en la germinación respecto al testigo en $15 \%$; estos resultados son similares a los encontrados por Zheng en el año 2000, quien evaluó 3 cepas diferentes ( $T$. viride, T. koningii, T. harzianum) para la germinación de la arveja en la Universidad de Massachusetts; sin embargo, son inferiores a los reportados por Hinojosa (16) en semillas de maracuyá (Passiflora edulisvar, Flavicar padegener), quien encontró aumento en la germinación hasta del $40 \%$. Este incremento en germinación puede ser muy representativo para los agricultores, debido a que eleva el número de plantas emergidas en $15 \%$, lo que representa mayor uniformidad en el cultivo, generando mayor eficiencia en las labores agronómicas y, por tanto, un aumento en la productividad.

Peso fresco parte aérea. En la Figura 1 se destaca la diferencia existente en el peso fresco de la parte aérea del T12, el cual es un $77 \%$ superior al del testigo. La variable mostró incremento significativo respecto al testigo cuando las plantas de arveja fueron sometidas a T12 y T10. Los resultados anteriores concuerdan con lo reportado en cultivos de papa (Solanum tuberosum) (17), melón (Cucumis Melo) (18) y aguacate (Persea americana L) (19); sin embargo, el incremento en el peso fresco de las plantas de arveja fue superior a todos estos cultivos, por lo que la arveja en comparación tiene una reacción favorable a la aplicación de Trichoderma.

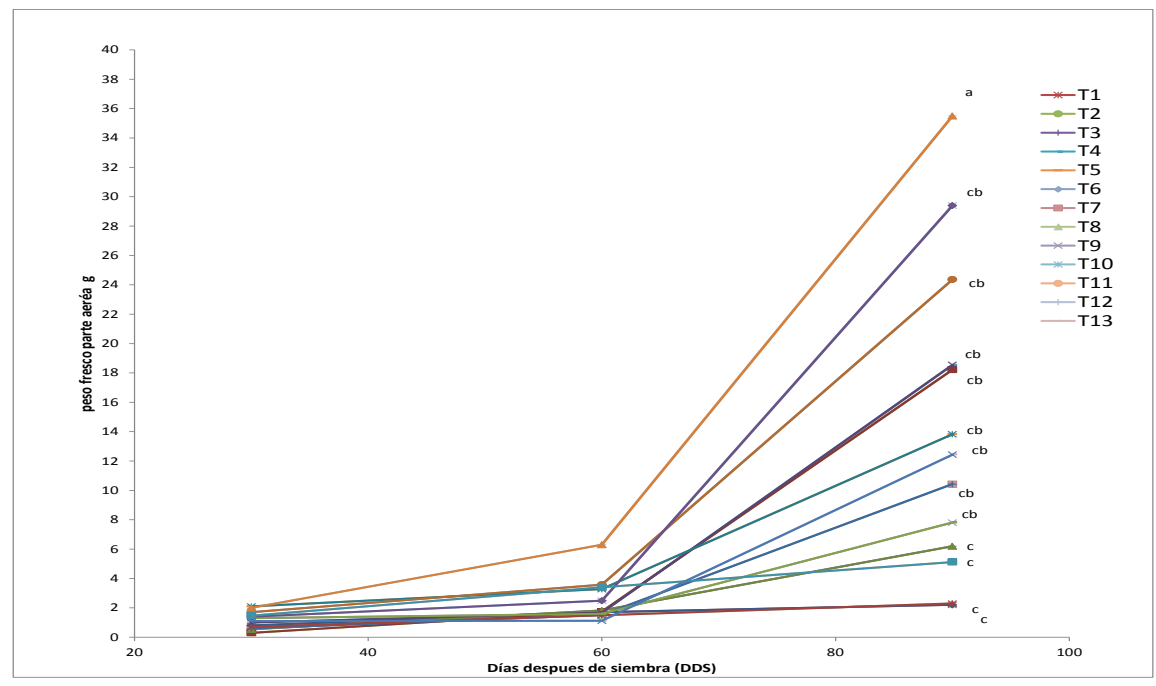

Figura 1. Peso fresco de la parte aérea de las plantas de arveja bajo la influencia de los tratamientos, semestre B 2013

Letras diferentes al final de las curvas indican diferencia estadística significativa $(p>0.05)$

Área foliar. En la Figura 2 se destaca la diferencia existente en el área foliar de las plantas sometidas a T11, que aumenta en $45 \%$ respecto al testigo. El área foliar de las plantas de arveja mostró un incremento significativo respecto al testigo cuando las plantas de arveja fueron sometidas a T13, T12, T11, T10, T9, T6, T5, T4, T3 y T2, mientras que los demás tratamientos no tuvieron un efecto en el área foliar de la planta. Los resultados anteriores concuerdan con lo reportado por varios autores en cultivos de café (Coffea arabica) (20), higo blanco (Picuscarica L.) (21), melón (Cucumis melo) (18) y aguacate (Persea americana L) (19); sin embargo, el incremento en el área foliar de las plantas de arveja fue inferior al café, pero superior a todos los demás cultivos, por lo que la arveja en comparación tiene una reacción intermedia a la aplicación de Trichoderma. 


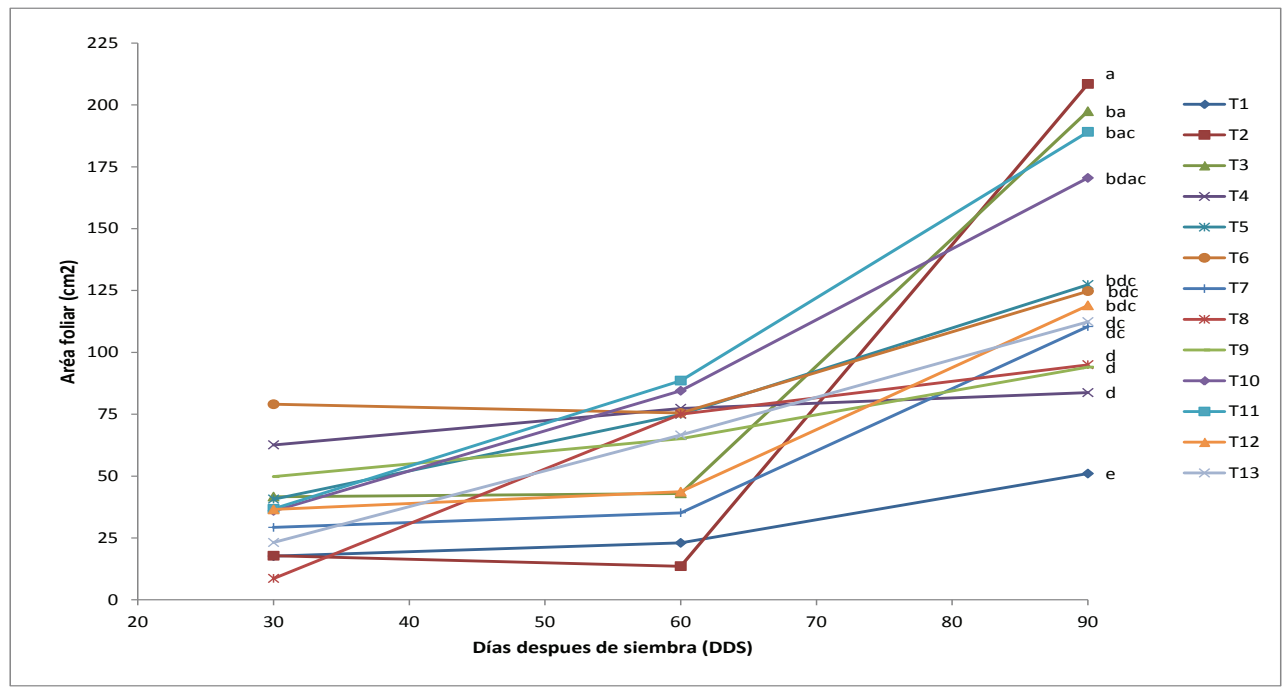

Figura 2. Área foliar de las plantas de arveja bajo la influencia de los tratamientos, semestre B 2013 Letras diferentes al final de las curvas indican diferencia estadística significativa $(p>0.05)$

\section{Incidencia de enfermedades}

- Ascochita pisi Lib. En todas las plantas de cada uno de los tratamiento hubo presencia de mancha ascochita (Ascochita pisi Lib.), lo que demuestra que Trichoderma sp. no genera un control efectivo para este patógeno.

- Fusarium sp. Debido a que no se realizó inoculación con ningún patógeno de suelo no se presentaron síntomas que evidenciaran la presencia de este patógeno en ninguno de los tratamientos, por lo que no se puede concluir la existencia de algún antagonismo con este patógeno.

Masa seca parte aérea. En la Figura 3 se destaca la diferencia del peso seco de la parte aérea de las plantas sometidas a T12, el cual es superior en un $92 \%$ respecto al testigo. El peso seco de la parte aérea de las plantas de arveja mostró un incremento significativo respecto al testigo cuando las plantas de arveja fueron sometidas a T12, T11, T10, T8, T6, T5, T3 y T2, mientras que las demás concentraciones no tuvieron un efecto en el peso seco de la parte aérea de la planta. Los resultados anteriores concuerdan con lo reportado por varios autores en cultivos de Tomate (Lycopersicum esculentum L.), pepino (Cucumis sativus Mill.) pimiento (Capsicu mannumm L.) (21) y maíz (Zea mays L.) (22); sin embargo, el incremento en el peso seco de la parte aérea de las plantas de arveja fue superior a todos estos cultivos, por lo que la arveja en comparación tiene una reacción más favorable a la aplicación de Trichoderma que estos cultivos. 


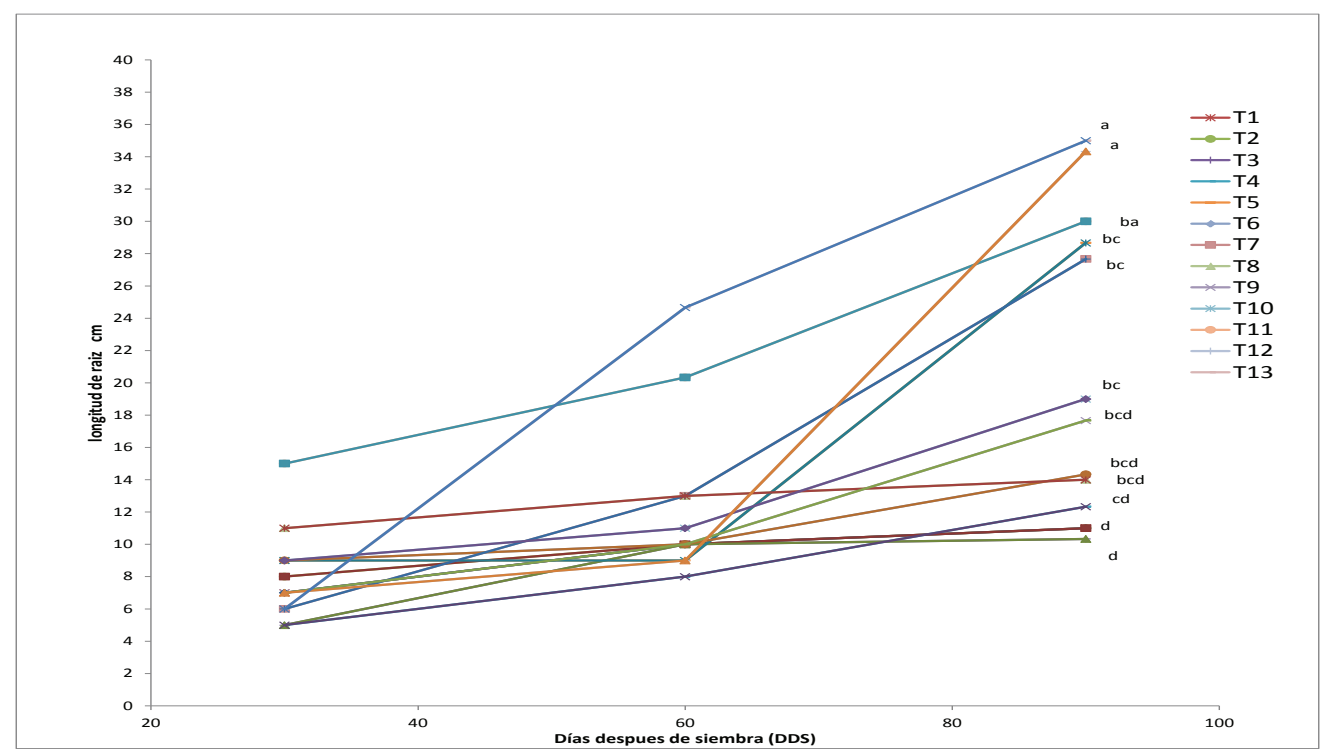

Figura 3. Peso seco de la parte aérea de las plantas de arveja bajo la influencia de los tratamientos, semestre B 2013.

Letras diferentes al final de las curvas indican diferencia estadística significativa ( $p>0.05)$

Longitud de raíces. En la Figura 4 se destaca la diferencia existente en la longitud radicular de T13 y T12, siendo significativamente superiores al testigo sin inocular en $21 \%$. La longitud de raíces de las plantas de arveja mostró incremento significativo respecto al testigo cuando fueron sometidas a T13 y T12, mientras que las demás concentraciones no tuvieron un efecto en la longitud de la raíz de la planta. Los resultados anteriores concuerdan con lo reportado por varios autores en cultivos de maíz (Zea mays) (12), maracuyá (Passiflora edulis var Flavicar padegener) (16), ají (Capsicu mannum var. longum) (18) y tomate (Lycopersicon esculentum L.) (23); sin embargo, en maracuyá el efecto del Trichoderma fue mayor cuando se aplicó la cepa nativa (16), mientras que en tomate las diferencias respecto al testigo sin inocular no fueron significativas (23), por lo que la arveja en comparación tiene una reacción favorable a la aplicación de Trichoderma.

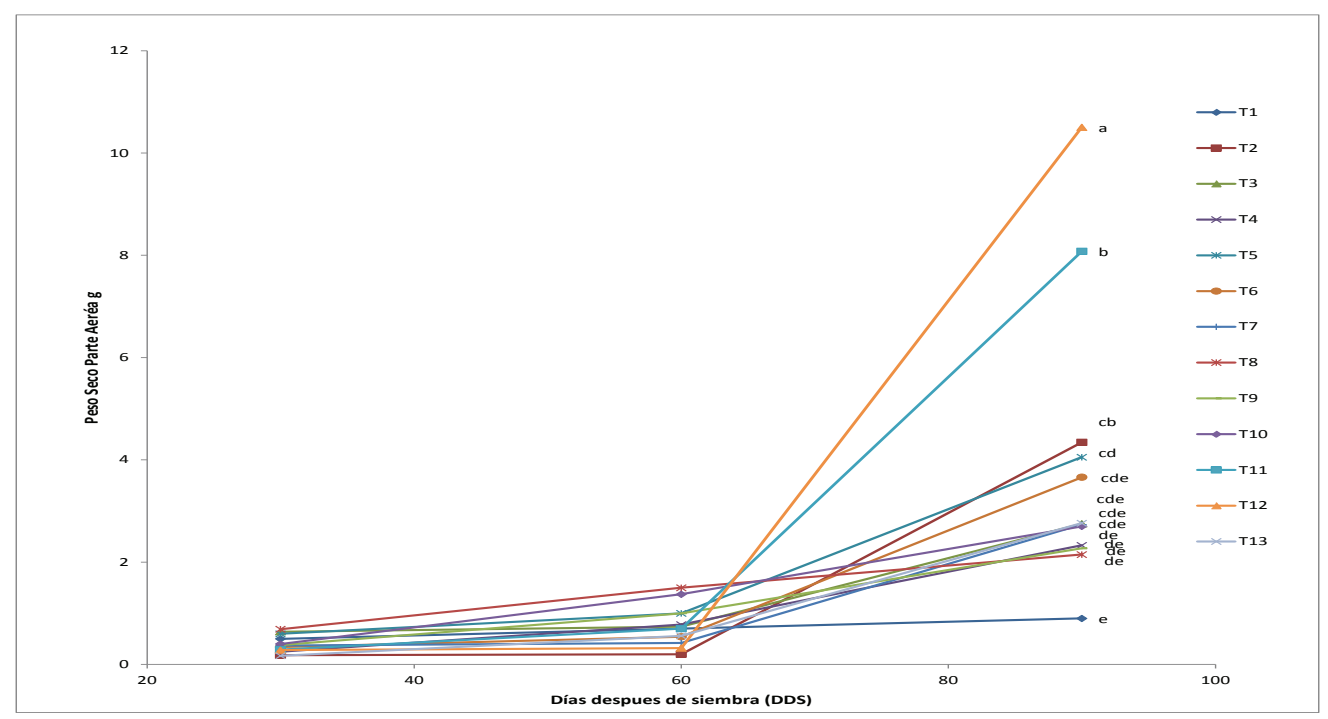

Figura 4. Longitud radicular de las plantas de arveja bajo la influencia de los tratamientos, semestre B 2013

Letras diferentes al final de las curvas indican diferencia estadística significativa $(p>0.05)$ 
Masa fresca raíz. En la Figura 5 se destaca la diferencia existente en el peso fresco radicular de las plantas sometidas a los tratamientos $\mathrm{T} 12, \mathrm{~T} 13$, T5 y T6, que generan un incremento significativo del peso fresco de la parte aérea respecto al testigo. Además, se destaca el incremento que genera el T12, ya que aumenta en más de un $65 \%$ el peso fresco de la raíz de las plantas de arveja. Las demás concentraciones no tuvieron un efecto en el peso fresco de la raíz de la planta. Los resultados anteriores concuerdan con lo reportado por varios autores en cultivos de fresa chilena
(Fragaria chiloensis L.) (11), tomate (Lycopersicum esculentum L.), pepino (Cucumis sativus Mill.) y pimiento (Capsicu mannumm L.) (21). Al comparar estos ensayos con los resultados obtenidos, el incremento en el peso fresco radicular de la arveja fue menor que en fresa chilena, pero muy superior al obtenido en tomate, pepino y pimiento, por lo que el peso fresco de la raíz de la arveja tiene una reacción significativa a la aplicación de Trichoderma.

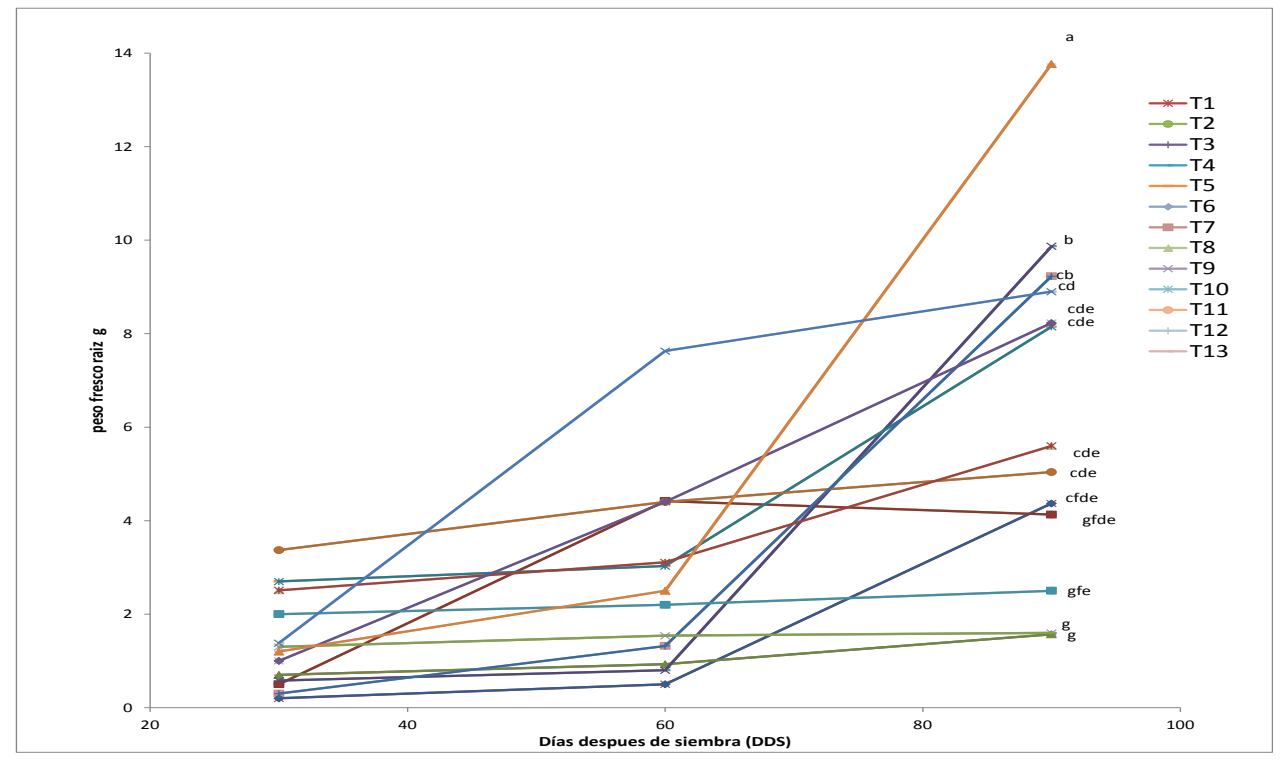

Figura 5. Peso fresco radicular de las plantas de arveja bajo la influencia de los tratamientos, semestre B 2013

Letras diferentes al final de las curvas indican diferencia estadística significativa $(p>0.05)$

Peso seco raíz. En la Figura 6 se destaca la diferencia existente en el peso seco de la raíz del T12, el cual es un $68 \%$ superior testigo. El peso seco de la raíz de las plantas de arveja mostró un incremento significativo respecto al testigo cuando las plantas de arveja fueron sometidas a T13 y T12. Las demás concentraciones no tuvieron un efecto en el peso fresco de la raíz de la planta. Los resultados anteriores concuerdan con lo reportado por varios autores en cultivos de pimiento (Capsicum annuum L.) (12), cebolla (Allium cepa L.) (10) y acelga (Beta vulgaris var. Cicla) (18); sin embargo, el incremento en el peso seco de la raíz de las plantas de arveja fue superior al mostrado por los cultivos de ají, pimiento y cebolla, pero inferior al mostrado por la acelga, por lo que la arveja en comparación tiene una reacción moderada a la aplicación de Trichoderma. 


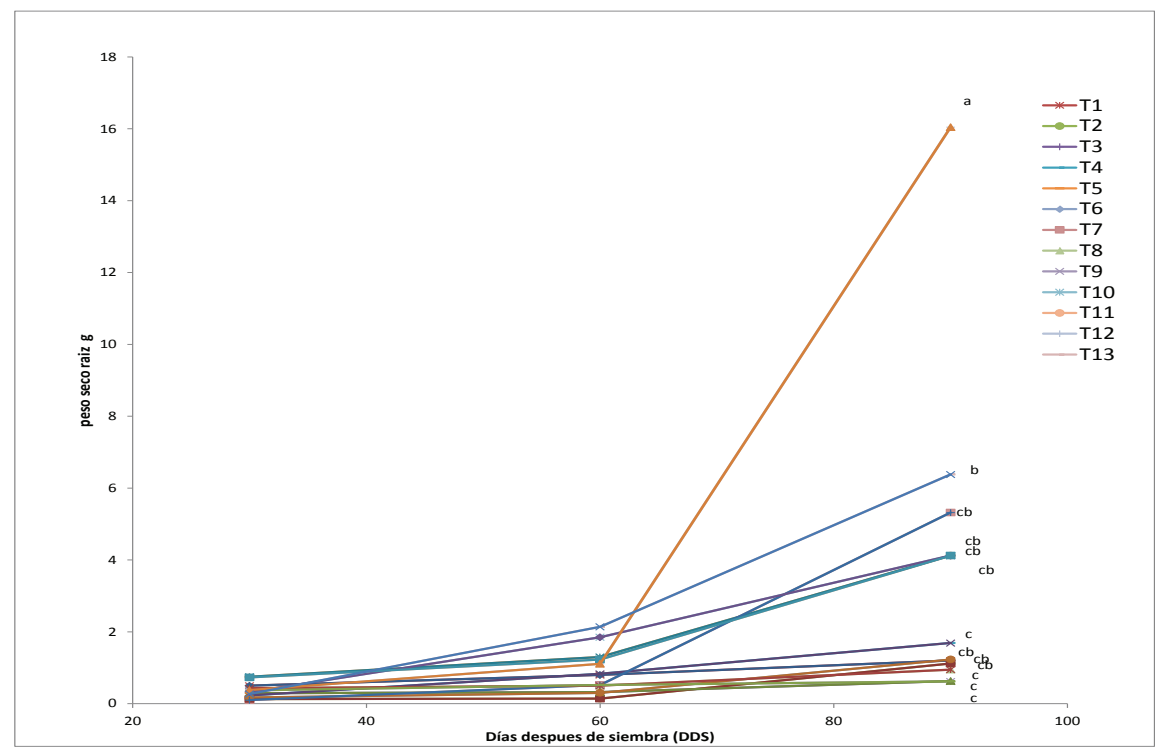

Figura 6. Peso seco de la raíz de las plantas de arveja bajo la influencia de los tratamientos, semestre B, 2013

Letras diferentes al final de las curvas indican diferencia estadística significativa $(p>0.05)$

\section{CONCLUSIÓN}

La aplicación de Trichoderma sp. comercial en el cultivo de arveja mejora notablemente el crecimiento y el desarrollo, influyendo en variables fisiológicas como germinación, área foliar, peso seco de la raíz, peso fresco de la raíz, peso seco de la parte aérea, peso fresco de la parte aérea y longitud de raíz, favoreciendo el rendimiento productivo del cultivo.

\section{REFERENCIAS}

(1) Fenalce (Federación Nacional de Cultivadores de Cereales y Leguminosas). Importancia de los cultivos representados por Fenalce. Colombia. 2010.

(2) Herrera-Cid RA. Control biológico de Rhizoctonia solani y Fusarium oxysporum en tomate bajo invernadero. Trabajo de grado, Facultad de Ciencias Agronómicas, Universidad de Chile. 2013.

(3) Corporación Colombia Internacional. CCl. Manual del exportador de frutas, hortalizas y tubérculos en Colombia. 2013.
(4) Pacheco C, Vergara C, Ligarreto G. Clasificación de 42 líneas mejoradas de arveja (Pisum sativum L.) por caracteres morfológicos y comportamiento agronómico. Revista Facultad Nacional de Agronomía de Medellín. 11 p. 2010.

(5) Ezziyyani M, Pérez C, Sid A, Requena M, Candela M. Trichoderma harzianum como biofungicida para el biocontrol de Phytophthora capsici en plantas de pimiento (Capsicum annuum L.). Tesis de Maestría, Departamento de Biología Vegetal, Facultad de Biología, Universidad de Murcia. Murcia, España. 2004.

(6) Howell R. Mechanisms employed by Trichoderma species in the biological control of plant diseases: The history and evolution of current concepts. 2003.

(7) Godes A. Perspectivas de los inoculantes fúngicos en Argentina. 2007.

(8) Valencia H, Sánchez J, Vera N, Valero M. Microorganismos solubilizadores de fosfatos y bacterias fijadoras de nitrógeno en páramos y región cálida tropical. 2007.

(9) Valero N. Determinación del valor fertilizante de microorganismos solubilizadores de fosfato en cultivos de arroz. Trabajo de grado, Universidad Nacional de Colombia. 2007. 
(10) Vera D, Pérez H, Valencia H. Aislamiento de hongos solubilizadores de fosfatos de la rizosfera del arazá (Eugenia stipitata, Myrtaceae). 2002.

(11) Altomare C, Norvell A, Björkman T, Harman G. Solubilization of phosphates and micronutrients by the plant-growth-promoting and biocontrol fungus Trichoderma harzianum Rifai. 1999.

(12) Börkman T, Blanchard L, Harman G. Growth enhancement of shrunken-2 sweet corn by Trichoderma harzianum: effect of environmental stress. J. Amer. Soc. Hort. Sci 1998; 23(1): 295-322.

(13) Dandurand L, Knudsen G. Influence of Pseudomonas fluorescent on hyphal growth and biocontrol activity of Trichoderma harzianum in the spermosphere and rhizosphere of pea. 1993.

(14) Cervantes L, Moreno J, Pulido A, Ceceña C, González D, Juárez O, Gutiérrez A. Uso de Trichoderma sp. para el biocontrol de la pudrición radicular en cebolla. 2009.

(15) Lara D. Uso de bacterias endofíticas para el control biológico del Mal de Panamá (Fusarium oxysporum f. sp. cubense) en el cultivar Gros Michel (AAA). Centro Agronómico Tropical de Enseñanza e Investigación. Turrialba, Costa Rica. 2009.

(16) Hinojosa J, Valero N, Mejía L. Trichoderma harzianum como promotor del crecimiento vegetal del maracuyá (Passiflora edulis var. Flavicarpa Degener) Departamento de Microbiología, Universidad Popular del Cesar. Valledupar, Colombia. 2008.

(17) Hoyos L, Villegas S, Peralta S. Evaluación de Trichoderma asperellum como biorregulador de Spongospora subterranea F. sp. subterranea. Trabajo de grado, Facultad de Ciencias Agropecuarias. Universidad Nacional de Colombia. 2008.

(18) Martínez A, Roldán A, Lloret E, Pascual J. Formulación de Trichoderma harzianum Rifai en la producción ecológica de plántulas de melón en semillero para el control de la fusariosis vascular. Dpto de Conservación de Suelos y Agua y Manejo de Residuos Orgánicos. Campus Universitario de Espinardo. Murcia, España. 2005.

(19) Benavides M, Sáenz E. Efecto de hongos benéficos sobre la nutrición y sanidad de aguacate. Corporación Colombiana de Investigación Agropecuaria. 2004.

(20) Santana R, Arbeláez A, Esmori C. Efecto de Trichoderma viride Rifai en el desarrollo de los injertos hipocotiledonares de café. Centro de Investigaciones Agropecuarias. Facultad de Ciencias Agropecuarias. Manica, Nicaragua. 2010.

(21) Galeano M, Méndez F, Urbaneja A. Efecto de Trichoderma harzianum Rifai (cepa t-22) sobre cultivos hortícolas. Departamento I+D. Koppert Biological Systems. 2009.

(22) Parra J, Pavone D. Efecto de Trichoderma spp. sobre Rhizoctonia solani y algunos parámetros fisiológicos en Zea mays L. bajo condiciones de vivero. Trabajo de grado, Laboratorio de Biotecnología Aplicada, Departamento de Biología, Facultad de Ciencias y Tecnología, Universidad de Carabobo. 2012.

(23) Jiménez C, Sanabria de Albarracin N, Altuna G, Alcano M. Efecto de Trichoderma harzianum (Rifai) sobre el crecimiento de plantas de tomate (Lycopersicon esculentum L.). Instituto Nacional de Investigaciones Agrícolas. Caracas, Venezuela. 2011.

(24) Gutiérrez J, Romero A, Kubiak D, Pezuk D, Bulak K, Cabral J. Utilización de un bioproducto a base de Trichoderma spp. como potenciador de crecimiento: Experiencia con horticultores del SACRA (Sindicato de Amas de Casa de la República Argentina) en almácigos de acelga (Beta vulgaris var. Cicla) y lechuga (Lactuca sativa). 2010. 